\title{
Analyses of murine GBP homology clusters based on in silico, in vitro and in vivo studies
}

\author{
Alexandra Kresse ${ }^{\dagger}$, Carolin Konermann ${ }^{\dagger}$, Daniel Degrandi, Cornelia Beuter- \\ Gunia, Jan Wuerthner, Klaus Pfeffer* and Sandra Beer*
}

\author{
Address: Institute of Medical Microbiology and Hospital Hygiene, Heinrich-Heine-University, Universitaetsstrasse 1, 40225 Duesseldorf, Germany \\ Email: Alexandra Kresse - alexandra.kresse@web.de; Carolin Konermann - konerman@uni-duesseldorf.de; \\ Daniel Degrandi - daniel.degrandi@uni-duesseldorf.de; Cornelia Beuter-Gunia - cornelia.beuter@uni-duesseldorf.de; \\ Jan Wuerthner - jan.wuerthner@gmx.de; Klaus Pfeffer* - klaus.pfeffer@uni-duesseldorf.de; Sandra Beer* - sandra.beer@uni-duesseldorf.de \\ * Corresponding authors †Equal contributors
}

Published: 10 April 2008

BMC Genomics 2008, 9:158 doi:10.1186/147|-2164-9-158

This article is available from: http://www.biomedcentral.com//47I-2/64/9/158

(C) 2008 Kresse et al; licensee BioMed Central Ltd.

This is an Open Access article distributed under the terms of the Creative Commons Attribution License (http://creativecommons.org/licenses/by/2.0), which permits unrestricted use, distribution, and reproduction in any medium, provided the original work is properly cited.

\begin{abstract}
The interactions between pathogens and hosts lead to a massive upregulation of antimicrobial host effector molecules. Among these, the $65 \mathrm{kDa}$ guanylate binding proteins (GBPs) are interesting candidates as intricate components of the host effector molecule repertoire. Members of the GBP family are highly conserved in vertebrates. Previous reports indicate an antiviral activity of human GBPI (hGBPI) and murine GBP2 (mGBP2). We recently demonstrated that distinct murine GBP (mGBP) family members are highly upregulated upon Toxoplasma gondii infection and localize around the intracellular protozoa $T$. gondii. Moreover, we characterised five new mGBP family members within the murine $65 \mathrm{kDa}$ GBP family. Here, we identified a new mGBP locus named $m G b p / I$. Based on bacterial artificial chromosome (BAC), expressed sequence tag (EST), and RTPCR analyses this study provides a detailed insight into the genomic localization and organization of the mGBPs. These analyses revealed a 166-kb spanning region on chromosome 3 harboring five transcribed mGBPs (mGbpl, mGbp2, mGbp3, mGbp5, and mGbp7) and one pseudogene (pseudomGbpl), as well as a 332-kb spanning region on chromosome 5 consisting of six transcribed mGBPs ( $m G b p 4, m G b p 6, m G b p 8, m G b p 9, m G b p / 0$, and $m G b p / l)$, and one pseudogene (pseudomgbp2). Besides the strikingly high homology of $65 \%$ to $98 \%$ within the coding sequences, the mGBPs on chromosome 5 cluster also exhibit a highly homologous exon-intron structure whereas the mGBP on chromosome 3 reveals a more divergent exon-intron structure. This study details the comprehensive genomic organization of mGBPs and suggests that a continuously changing microbial environment has exerted evolutionary pressure on this gene family leading to multiple gene amplifications. A list of links for this article can be found in the Availability and requirements section.
\end{abstract}

\section{Background}

The guanylate binding proteins (GBPs) were first described in 1979 when Gupta et al. identified a $67 \mathrm{kDa}$ protein induced in human fibroblasts after interferon $\gamma$
(IFN $\gamma$ ) stimulation [1]. Some years later, it was shown that two orthologous proteins are expressed in murine fibroblasts after stimulation with IFN $\gamma$ [2]. Besides the strong inducer IFN $\gamma$, the GBPs can also be induced by type I inter- 
ferons [2-6], tumor-necrosis-factor $\alpha$ (TNF- $\alpha$ ), interleukin-1 $\beta$ (IL-1 $\beta)$, IL-1 $\alpha$ [7,8], and TLR agonists [5].

Human and murine GBPs possess the unique ability to bind to agarose-immobilized GMP (guanosine monophosphate), GDP (guanosine diphosphate), and GTP (guanosine triphosphate) with the same affinity, thereby differing from heterotrimeric or Ras-like GTP-binding proteins [2]. In addition, they hydrolyse GTP not only to GDP but also to GMP [9]. Further biochemical properties of the GBPs are the low binding affinity to nucleotides, their stability in the absence of guanine nucleotides and their high turnover GTPase activity [10]. Remarkably, the sequence of the common G4-motif N/TKxD is modified in the GBPs to the unique T(L/V)RD motif [10]. In the case of hGBP1 a nucleotide-dependent oligomerization and concentration-dependent GTPase activity has been observed [11]. These biochemical properties classified the GBPs as distantly related family members of the dynamin superfamily despite the lack of any sequence homology of the primary sequences $[11,12]$. The similarity to the dynamin family is further corroborated by the analysis of the crystal structure of hGBP1. It has an amino-terminal globular domain containing the GTP binding region and an elongated carboxy-terminal series of $\alpha$-helices. The GBPs possess the common 'dynamin domain structure' with a GTPase domain ( 300 residues), a 'middle' or 'assembly' domain (150-200 residues) and a GTPase effector domain ( $\sim 100$ residues) [11].

Although, the GBPs have been discovered almost 30 years ago, only little is known about their biological function. It has been suggested that the GBPs are important for cell growth regulation as demonstrated for mGBP2 and hGBP1 [7,13]. Both, hGBP1 and mGBP2 also alter matrix metalloproteinase (MMP) gene expression and thereby change cellular interactions with the extracellular environment [14]. In addition, hGBP1 was reported to be involved with paclitaxel resistance in ovarian cancer cell lines [15]. Further studies revealed that hGBP1 and mGBP2 exhibit a moderate antiviral activity against vesicular stomatitis virus (VSV) and encephalomyocarditis virus (EMCV) $[16,17]$. Recently, we have demonstrated that mGBPs are highly upregulated in mice after infection with Listeria monocytogenes or Toxoplasma gondii, and localize around the parasitophorous vacuole of $T$. gondii, thus suggesting that the mGBPs play a role in the defense against intracellular bacteria and apicomplexa [5].

Besides humans and mice the GBPs have been found in rats [18], chicken [19], fish [20], and several other vertebrate species. In humans seven orthologs and at least one pseudogene have been identified [21,22]. In mice five GBPs have been described $[2,23,24]$. Recently, one additional mGBP was discovered by an in silico study [21]. In search of new IFN $\gamma$ regulated genes using Affymetrix analyses we independently identified $m G b p 6, m G b p 7$, and $m G b p 8$ [5]. Further comprehensive genome and sequence analyses yielded two more homologous genes, named $m G b p 9$ and $m G b p 10$ [5]. The subsequent investigation of mGBP gene loci revealed an incorrect assembly concerning the $m G b p 8$ locus in the genome databases (Ensembl, NCBI). Thus, to clarify the genomic organization of mGBP coding genes, we used BAC and EST sequences obtained from NCBI. Based on these sequence analyses we were able to identify another mGBP locus named $m G b p 11$. In this study, we address the genomic organization and localization of each mGBP family member and present a revised assembly of the murine GBP homology clusters on chromosomes 3 and 5 .

\section{Results \\ The $m$ GBP genes are arranged in two clusters located on chromosomes 3 and 5}

Although the first guanylate binding protein had been described almost 30 years ago further family members have been discovered lately. A recent in silico study has proposed a number of six mGBPs and three pseudogenes [21]. We recently raised the number up to ten mGBP members [5]. In this study, we performed extensive homology searches on the whole genome to identify further mGBP loci. Single exons of known mGBPs were used for homology searches against murine genome databases using the BlastN algorithm [25]. Thereby, another mGBP family member was discovered, now designated mGBP11 (Acc. No. EU304258). The $m G b p 11$ locus was confirmed by BAC analyses. Hence, we extend the family to eleven mGBPs and two pseudogenes which all cluster on two chromosomes. One gene cluster is located on chromosome 3 within the $\mathrm{H} 3$ region and the second gene cluster is found within the E5 region on chromosome 5 (Fig. 1A). For further analysis of these two gene clusters we used BAC sequences obtained from NCBI. On chromosome 3 the BACs RP23-100J23 and RP24-314I8 cover the respective chromosomal regions between $142.44 \mathrm{MB}$ and $142.60 \mathrm{MB}$ harboring $m G b p 1, m G b p 2, m G b p 3, m G b p 5$, $m G b p 7$, and a pseudogene named pseudom $G b p 1$ (Fig. 1B). This gene cluster spans approximately $166 \mathrm{~kb}$. The length of each gene locus ranges from $3.7 \mathrm{~kb}$ (pseudom $\mathrm{Gbp} 1$ ) to $34.9 \mathrm{~kb}(m G b p 1)$. The mGBPs located on chromosome 3 are all transcribed from the positive strand.

Further, we used the BACs RP24-63G23, RP23-329M7 and RP24-210D14 for analyses of the region between 105.25 $\mathrm{MB}$ and 105.58 $\mathrm{MB}$ on chromosome 5 (Fig. 1C). By means of these BAC sequences we were able to determine the precise loci for $m G b p 4, m G b p 6$ (formerly mpa2l), $m G b p 8, m G b p 9, m G b p 10, m G b p 11$, and the pseudogene pseudomGbp2. This cluster has approximately twice the size of the cluster on chromosome 3 with an extension of 

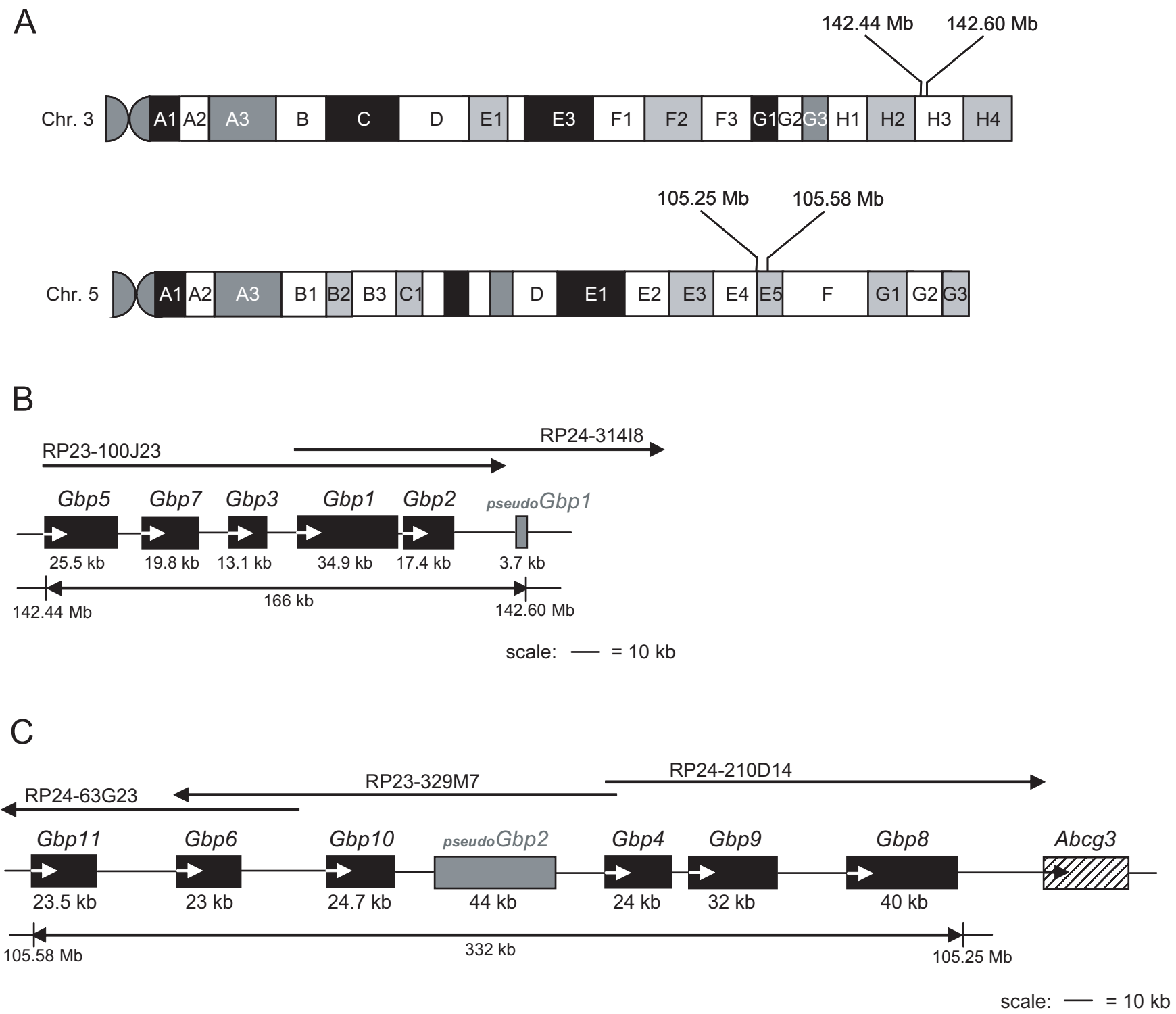

Figure I

mGBP genes cluster on chromosomes 3 and 5. A. One mGBP cluster is located within the $\mathrm{H} 3$ region on chromosome 3 (I42.44 MB to I42.60 MB) and the other within the E5 region on chromosome 5 (I05.25 MB to I05.58 MB). Genomic organization of the mGBP genes clustered on chromosome 3 (B) and chromosome 5 (C). Each gene locus is illustrated as a black rectangle, pseudogenes are shown in grey. The locus of the flanking gene Abcg3 is shaded. The approximate length of each gene is depicted below the corresponding rectangle. Transcriptional direction is indicated by white arrows. The approximate length of each cluster is shown at the bottom of the respective figure. The BACs used in this study are stated above the corresponding region of the chromosomes. The scale bar equals a length of $10 \mathrm{~kb}$.

$332 \mathrm{~kb}$. The length of each gene locus on chromosome 5 ranges from $23 \mathrm{~kb}(m G b p 6)$ to $44 \mathrm{~kb}$ (pseudomGbp2). In contrast to the mGBPs located on chromosome 3 the mGBPs on chromosome 5 are all transcribed from the negative strand.

\section{Conserved exon-intron structure in the mGBPs}

Besides their clustering on two chromosomes, the mGBPs share a highly similar genomic structure (Fig. 2). All members of the $65 \mathrm{kDa}$ mGBP gene family consist of eleven exons, except $m G b p 8$ which lacks exon 6 . Since exon 6 is composed of 246 basepairs no frameshift is generated. 
A
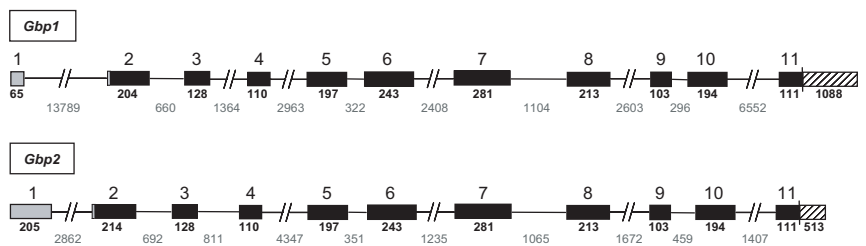

Gbp3
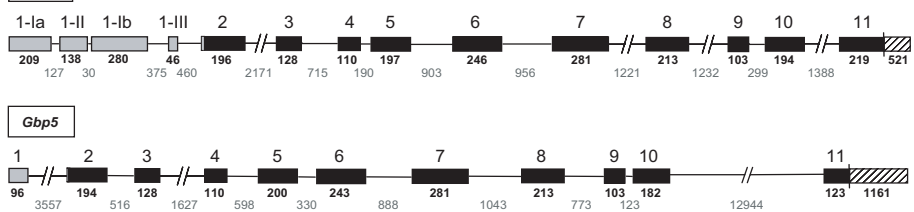

Gbp5a

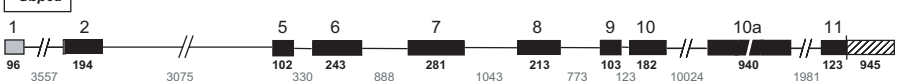

Gbp7

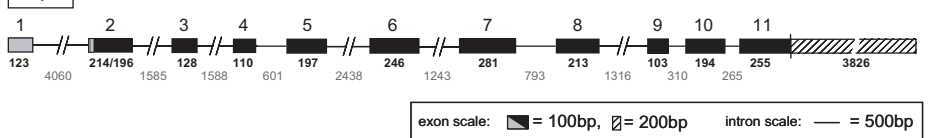

B

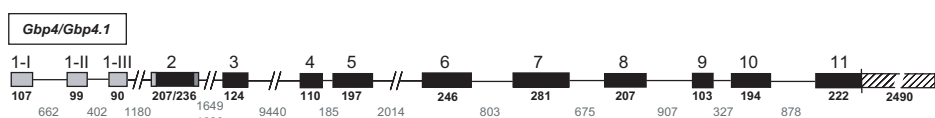

Gbp6

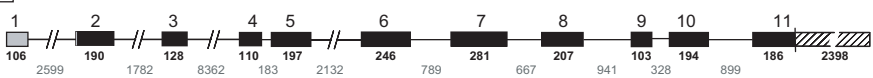

Gbp8
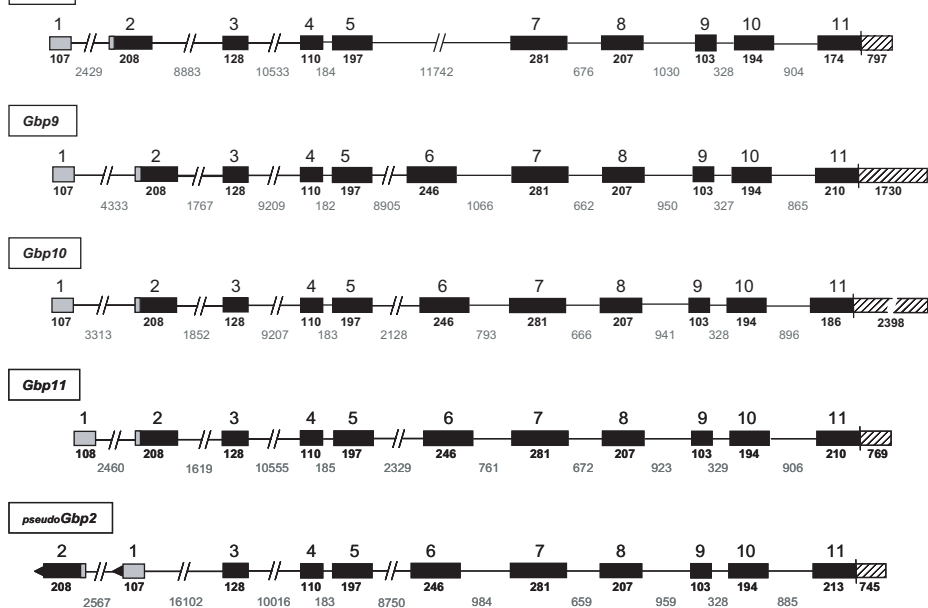

exon scale: $\boldsymbol{\nabla}=100 \mathrm{bp}, \boldsymbol{\nabla}=200 \mathrm{bp} \quad$ intron scale: $-=500 \mathrm{bp}$

\section{Figure 2}

Genomic organization of $\mathrm{mGBP}$ genes on chromosome 3 (A) and chromosome 5 (B). Translated exons are illustrated in black, 5' untranslated exons in grey and $3^{\prime}$ untranslated exons are shaded. The alternative 5 ' non-coding exons of mGBP3 (I-a, II, I-b, and III) and mGBP4 (I, II, and III) are numbered separately. The mRNAs for mGBP5 and mGBP5a are differentially spliced transcripts encoded by the same genomic locus. However, since alternative splice acceptor sites in exon 5 are used and an alternative exon 10a is inserted in the different mRNAs two schematic illustrations are provided. The inversion of the first two exons of pseudogene pseudomGbp2 is indicated by black arrowheads. The length (bp) of each exon and intron is specified. The depicted scale bars correspond to the different rulers used for introns, coding or non-coding exons. 
The cloning and sequencing of mGBP8 cDNA confirmed these genomic findings. Interestingly, the translation of all mGBPs starts within exon 2.

The size of the first non-coding exon of the mGBPs on chromosome 3 ranges from $65 \mathrm{bp}$ (mGBP1) to $205 \mathrm{bp}$ (mGBP2) (Fig. 2A). For mGBP3 several alternative 5' UTR exons were identified. In the database 26 EST and cDNA sequences which cover the 5' UTR from mGBP3 were found: seven ESTs contain exon 1-Ia upstream of exon 2, three sequences harbor exon 1-Ia and 1-Ib in combination, 16 sequences comprise exon 1-II and only one EST was found having exon 1-III as a $5^{\prime}$ non coding exon. For mGBP7 two different splice sites in the 5' UTR of exon 2 were observed as reported previously [21]. In every mGBP locus the exons 3 (128 bp), exons 4 (110 bp), exons 5 (197 bp), exons 7 (281 bp), exons 8 (213 bp), exons 9 (103 bp), and exons 10 (194 bp) cover the same size, except mGBP5 which has an exon 5 with 200 bp instead of $197 \mathrm{bp}$ and an exon 10 with $182 \mathrm{bp}$ instead of $194 \mathrm{bp}$. Moreover, for mGBP5 an alternative splice form, mGBP5a, which lacks exons 3, exon 4, a part of exon 5, and possesses an exon 10a, has been described [24]. Exons 6 of mGBP1, mGBP2, and mGBP5 consist of 243 bp whereas exons 6 of the other mGBPs span 246 bp.

Interestingly, the mGBPs on chromosome 5 show even higher similarities concerning the genomic organisation (Fig. 2B). The first exons of these mGBPs have nearly identical sizes (between 106 and $108 \mathrm{bp}$ ). Only for mGBP4 alternative 5' non-coding exons were observed (1-I, 1-II, and 1-III). EST sequences harboring either exon 1-I or exon 1-II as well as all three alternative 5' UTR exons were found in the database. Furthermore, we were able to identify an alternative splice form of mGBP4. Due to a mutation at the splice donor site in intron 2 two different transcripts, named mGBP4 and mGBP4.1, are generated [26]. Except exon 3 of mGBP4, all mGBPs on chromosome 5 share identical sizes of the individual coding exons 3 to 10 . Even the intron sequences are highly conserved in these genes. In particular, intron 4, intron 7 , intron 8, intron 9, and intron 10 exhibit high similarities. Comparable to all other mGBPs the pseudogene pseudomGbp2 shows a highly conserved exon-intron structure. Yet, an inversion of 'exon 1' and 'exon 2' results in a non-functional locus. Taken together, due to their high structural homologies these loci seem to be duplicated quite recently.

\section{In vitro and in vivo analyses of the new mGBP members}

After determination of the precise exon-intron structure of each mGBP we confirmed the predicted sequences by cloning and sequencing the corresponding cDNAs out of IFN $\gamma$ stimulated macrophages. These studies revealed that mGBP11 has a premature stop codon within exon 8 lead- ing to an ORF sequence with only $1329 \mathrm{bp}$. The amino acid sequence and the GTP-binding motif are depicted in additional file 1.

In order to elucidate the basal expression and inducibility of the new mGBP family members RT-PCR analyses were performed. Therefore, we compared the expression levels in unstimulated and IFN $\gamma$ stimulated ANA-1 macrophages (Fig. 3A). The mGBPs were only detectable upon stimulation, except mGBP9, which was already expressed in unstimulated cells. Besides IFN $\gamma$ stimulation, the infection of $\mathrm{C} 57 \mathrm{BL} / 6$ mice with $L$. monocytogenes led to a rapid upregulation of all tested mGBPs in the liver (Fig. 3B). Interestingly, slight mGBP11 RNA expression was detectable in the liver of uninfected mice.

\section{Sequence alignments and homologies of the mGBPs}

Based on ORF sequences of the 11 mGBPs we accomplished a cDNA alignment using the ClustalW algorithm (Fig. 4 and additional file 2). As shown in the alignment, the mGBPs share a high degree of homology throughout their coding sequences whereas the 5 ' part is the most conserved region of the mGBPs. Especially, mGBP6 and mGBP10 are the most homologous mGBPs differing in only 30 basepairs within an ORF sequence of $1836 \mathrm{bp}$. The conserved 5' part codes for the four typical GTP binding motifs. Although there are some differences in the nucleotide sequence of the GTP binding motifs the amino acid sequences are almost identical (Table 1). In detail, the G1 motifs of mGBP2, mGBP3, mGBP5/5a, mGBP6, mGBP7, mGBP8, mGBP9, and mGBP11 are identical, only the G1 motifs of mGBP1 and mGBP10 differ in two amino acids, and the G1 motif of mGBP4/4.1 differ in three amino acids. The G2 and G3 motifs are the same among all mGBPs. Regarding the G4 domain the mGBPs can be divided into the two subgroups 'TVRD' (mGBP4.1, mGBP6, mGBP7, mGBP8, mGBP9, and mGBP11) and 'TLRD' (mGBP1, mGBP2, and mGBP5), except mGBP3 with a 'AVRD' and mGBP10 with a 'IVRD' motif (Table 1). Most likely the functional consequences are minor since the amino acid variations in different G4 motifs lead to conservative exchange of aliphatic $(\mathrm{L} / \mathrm{V})$ and hydrophobic (T/A/I) amino acids. Interestingly, the mGBPs from the 'TLRD' group possess a C-terminal CaaX motif, which can be modified by isoprenylation [27]. Overall, the 3' parts of the mGBPs are more divergent than the $5^{\prime}$ parts.

Using the maximum likelihood method we generated a phylogenetic tree of the mGBP family based on the ORF sequences (Fig. 5). This phylogenetic analysis revealed three predominant homology clusters. Two homology clusters are located on chromosome 3. The first homology cluster consists of mGbp1, mGbp2, and mGbp5/5a. The second homology cluster contains mGbp3 and mGbp7. The homologies of the members of these two clusters are 

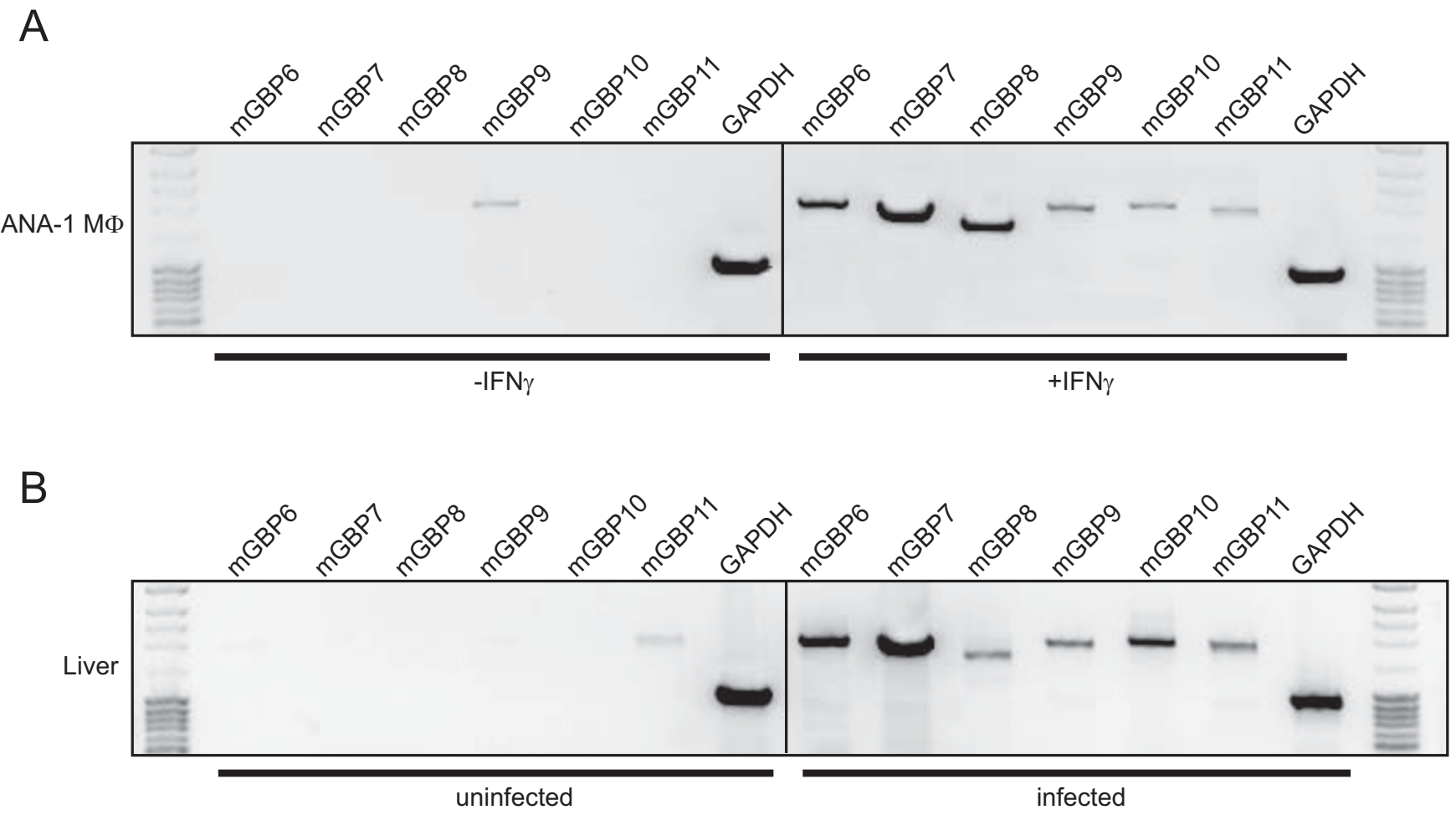

Figure 3

Transcriptional analyses of mGBP6, mGBP7, mGBP8, mGBP9, mGBP I 0, and mGBP I I. Amplification of mGBP6, mGBP7, mGBP8, mGBP9, mGBPI0, and mGBPI I using specific primers listed in additional file 3 and cDNAs derived from RNA of unstimulated and IFN $\gamma$ stimulated ANA-I macrophages (A), and RNA from liver of uninfected and $L$. monocytogenes infected C57BL/6 mice (B). GAPDH primers were used as an internal control.

Table I: Comparison of the conserved GI, G2, G3, and G4 motifs of the mGBPs. The canonical GTP motifs of all mGBPs and the overall consensus sequence are shown. Amino acid substitutions are highlighted in bold.

\begin{tabular}{lcccc}
\hline & GI & G2 & G3 & G4 \\
\hline mGBPI & GFYHTGKS & T & DTEG & TLRD \\
mGBP2 & GLYRTGKS & T & DTEG & TLRD \\
mGBP3 & GLYRTGKS & T & DTEG & AVRD \\
mGBP4 & GWSHTGKS & - & --- & --- \\
mGBP4.I & GWSHTGKS & - & DTEG & TVRD \\
mGBP5 & GLYRTGKS & T & DTEG & TLRD \\
mGBP5.a & GLYRTGKS & - & --- & TLRD \\
mGBP6 & GLYRTGKS & T & DTEG & TVRD \\
mGBP7 & GLYRTGKS & T & DTEG & TVRD \\
mGBP8 & GLYRTGKS & T & DTEG & TVRD \\
mGBP9 & GLYRTGKS & T & DTEG & TVRD \\
mGBPI0 & GWSRTGKS & T & DTEG & IVRD \\
mGBPI I & GLYRTGKS & T & DTEG & TVRD \\
\hline consensus & GxxxTGKS & T & DTEG & x(V/L)RD \\
\hline
\end{tabular}

lower than $60 \%$ (Table 2). The second cluster is with approximately $70 \%$ amino acid identities more closely related to the mGBPs in the third homology cluster on chromosome 5 encompassing mGbp4, mGbp6, mGbp8, mGbp9, mGbp10, and mGbp11. Within the cluster on chromosome 5 sequence identities reach $65 \%$ up to $98 \%$ (Table 2). The short branches implicate a just recent duplication of these genes on chromosome 5 .

\section{Discussion}

In order to find new IFN $\gamma$ regulated host effector molecules we were able to identify and characterize five novel members of the mGBP family [5]. Further, we showed that all hitherto identified members of the mGBP family are IFN $\gamma$ induced and moreover are highly upregulated in mice after infection with $L$. monocytogenes or T. gondii. Furthermore, we demonstrated that in infected cells most mGBPs surround the parasitophorous vacuole of $T$. gondii [5]. Consecutively, within this study, comprehensive homology and motif searches against public databases (NCBI, Ensembl) using EST and BAC sequences resolved 


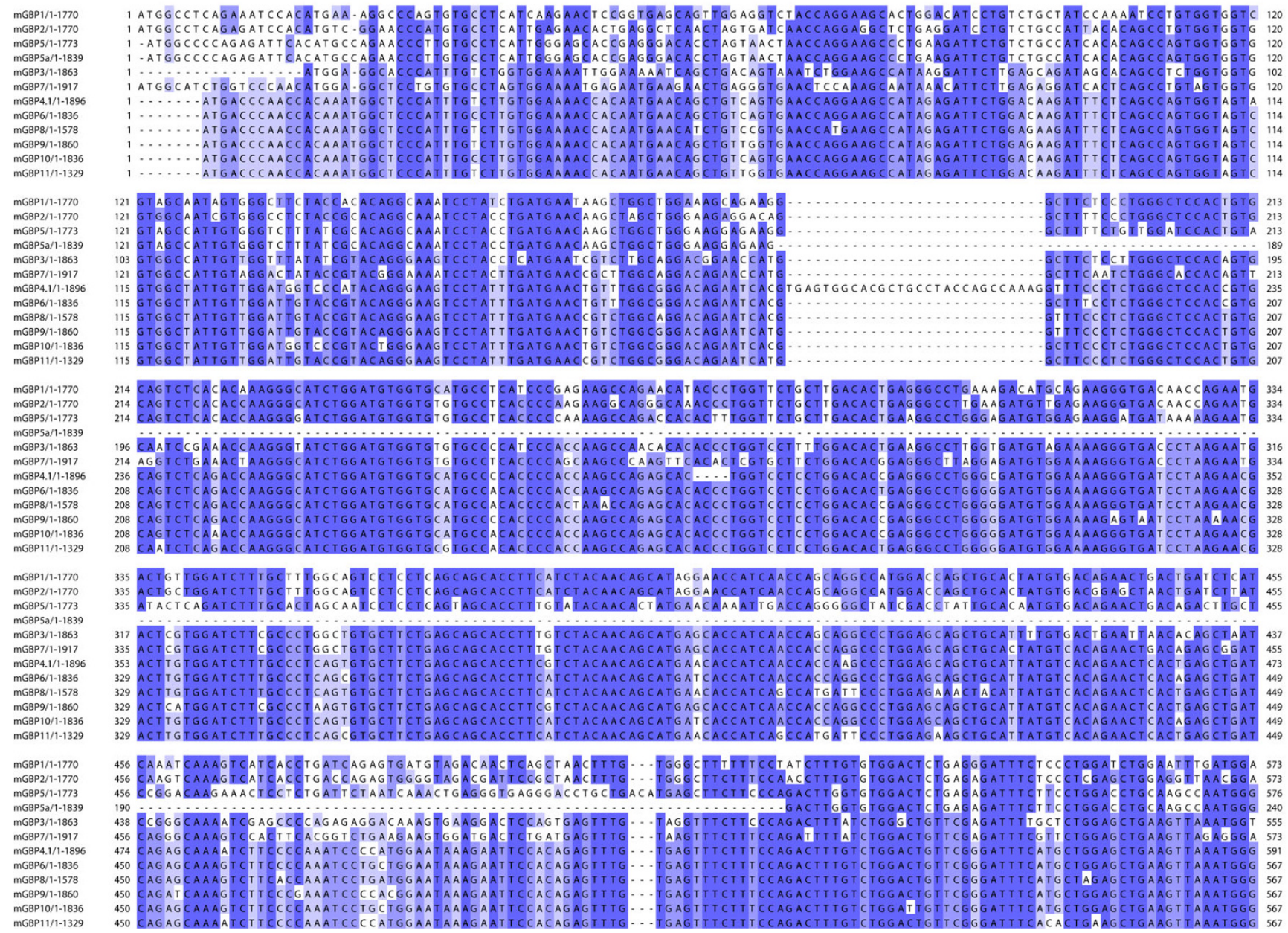

\section{Figure 4}

ClustalW alignment of ORF sequences of the eleven 65 kDa mGBP members. The alignment-layout was edited with the JalView software. Basepairs identical in all eleven mGBPs are marked in dark blue, identical in $\geq 75 \%$ in medium blue and identical in $\geq 50 \%$ in light blue. Red boxes highlight the conserved GTP binding motifs: P-loop (GI), Switch I (G2), Switch II (G3) and G4. This figure shows the upper quartile, for the full image please see additional file 2.

the genomic organization and localization of the mGBPs in more detail. During these analyses we were able to identify the additional mGBP member mGBP11. The scope of this study was to determine the precise loci of the 11 mGBPs and of the two pseudogenes, to compare the structure and organization of the mGBPs, to compile all cDNA sequences, and to verify the expression of mGBP mRNAs.

\section{Genomic organization of the $m$ GBPs}

The combined analyses revealed two mGBP homology clusters on chromosomes 3 and 5. One mGBP cluster is located within the $\mathrm{H} 3$ region on chromosome 3 which is in contrast to previously published data where the cluster was mapped to the $\mathrm{H} 1$ region [21]. The second cluster is located in the 55 region on chromosome 5 which is in accordance to Olszewski et al. [21]. Furthermore, Olsze- wski et al. noted that within the mGBP cluster on chromosome 5 the only functional mGBP gene is $m G b p 4$ and that in addition three pseudogenes (pseudomGbp2, pseudom $m b p 3$, and pseudom $\mathrm{Gbp} 4$ ) are located on this chromosome. Our in silico and mRNA sequence analyses now clearly demonstrate that besides $m G b p 4$ five expressed mGBPs ( $m G b p 6, m G b p 8, m G b p 9, m G b p 10$, and $m G b p 11$ ) are located on chromosome 5 (this study and [5]). In a previous report, we have shown that the $m G b p 4$ locus does not encode for a complete mGBP4 protein [26]. For pseudomGbp2 we could not find any corresponding EST sequence and were not able to clone a cDNA corresponding to pseudomGBP2, thus excluding the possibility of an alternative start downstream of exon 2. Based on BAC analyses we could demonstrate that the two pseudogenes pseudomGbp3 and pseudomGbp4 described by Olszewski et al. are both part of $m G b p 8$ 
which was virtually disrupted by the Abcg 3 gene locus in a former incorrect assembly within the public databases (see also [21]). Further transcript analyses revealed a functional $m G b p 8$ locus and showed an IFN $\gamma$ dependent upregulation of the mGBP8 mRNA comparable to mGBP6, mGBP9, mGBP10, and mGBP11. Similarly, all these mGBPs were highly induced upon $L$. monocytogenes infection in C57BL/6 mice (this study and [5]). The BAC sequences as well as our sequenced cDNA clones of the newly identified mGBP11 contain a premature stop codon within exon 8, leading to an ORF sequence of only $1329 \mathrm{bp}$. However, in the database (NCBI) one cDNA of

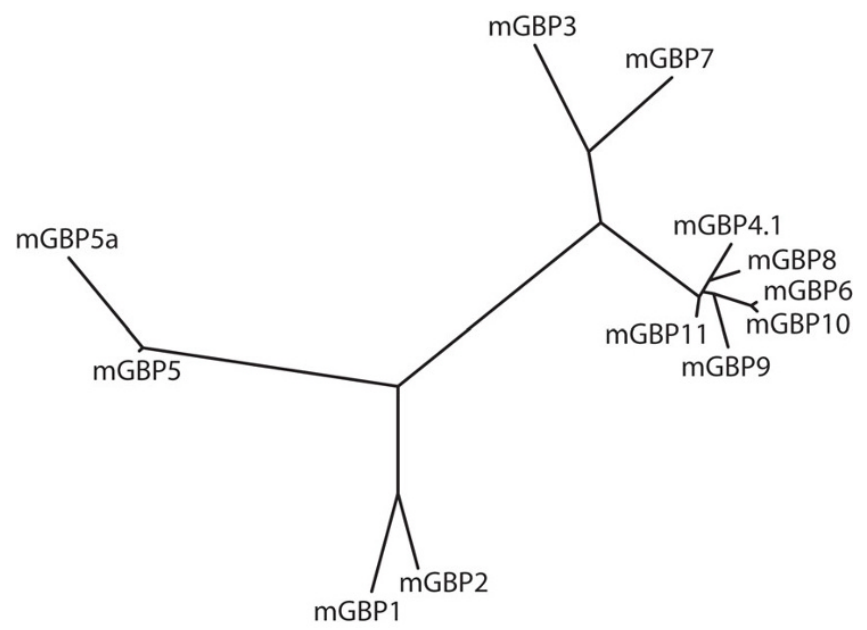

Figure 5

Phylogenetic tree of the mGBP cDNAs. The tree was created based on ORF sequences using the neighbor-joining method of the treepuzzle software. Branch lengths are measured relative to the estimated numbers of substitutions. Therefore, mGBP5 and mGBP5a appear less similar as they are actually on their amino acid level.
mGBP11 without a premature stop was found (Acc. No. $\mathrm{BC} 111039)$. It might be possible that the presence of different mGBP11 cDNAs is due to allelic variation. Further studies have to clarify, whether from this locus a functional protein can be translated and whether other mouse backgrounds differ in exon 8 .

In a recently published report, some subtle differences to our extensive genomic analyses have been described [28]. Firstly, in this report no mGBP7 gene is presented. Secondly, mGBP6 in [28] is termed mGBP7 based on our NCBI database submission in 2006 (BK005760, [5]). Thirdly, mGBP12 has been deposited in 2007 as mGBP11 by us (EU304258, this study). To keep consistency between database and nomenclature we propose to refer to the mGBP assignment in Figure 1. This is also in accordance with the extensive protein sequence and functional analyses which were provided previously [5]. mGBP13 has been described as a pseudogene by Olszewski and here (see Fig. 1 pseudoGbp1). Unfortunately, no description of the methods used for the identification of the mGBP13 locus is given in [28]. However, further studies are required to confirm whether this is a functional mGBP locus.

Besides the chromosomal localization of the mGBPs we also elucidated the exon-intron structure of these genes. Interestingly, all mGBPs consist of eleven exons with an impressively similar gene organization, with only one exception in $m G b p 8$ which lacks exon 6 . In addition, the translation of all mGBPs starts in exon 2. For $m G b p 3$ and $m \mathrm{Gbp} 4$ alternative non-coding exons were found in the $5^{\prime}$ regions. The usage of different 5 ' exons may influence the stability of the mRNAs [29]. Indeed, the frequencies of ESTs of mGBP3 and mGBP4 with the different alternative $5^{\prime}$ exons are quite variable, so their functional significance has to be validated. It has been reported that mRNAs of genes with alternative 5 ' coding or non-coding exons are

Table 2: Percent identities of the $m$ GBPs based on ORF sequences. For the calculation of percent identities a multiple sequence alignment with the ClustalW algorithm using MegAlign (DNAStar) was performed. Percent identities greater than $80 \%$ are shown in bold.

\begin{tabular}{|c|c|c|c|c|c|c|c|c|c|c|c|c|}
\hline \multicolumn{13}{|c|}{ Percent Identities } \\
\hline & mGBPI & mGBP2 & mGBP3 & mGBP4.I & mGBP5 & mGBP5.a & mGBP6 & mGBP7 & mGBP8 & mGBP9 & mGBPIO & mGBPI I \\
\hline mGBPI & ------ & 82.4 & 56.8 & 57.6 & 64.1 & 46.4 & 58.6 & 58.2 & 51.0 & 57.7 & 58.5 & 46.9 \\
\hline mGBP2 & & ----- & 58.9 & 58.5 & 64.9 & 45.8 & 59.3 & 58.7 & 51.9 & 58.7 & 59.0 & 46.7 \\
\hline mGBP3 & & & ----- & 69.6 & 52.8 & 40.0 & 69.8 & 78.2 & 61.0 & 69.7 & 69.8 & 53.8 \\
\hline mGBP4.I & & & & ----- & 55.3 & 40.4 & 86.9 & 69.4 & 76.8 & 87.0 & 86.8 & 66.1 \\
\hline mGBP5 & & & & & ----- & 71.2 & 55.4 & 55.6 & 47.9 & 54.5 & 54.9 & 45.6 \\
\hline mGBP5.a & & & & & & ------ & 39.3 & 40.5 & 32.3 & 39.5 & 39.0 & 29.3 \\
\hline mGBP6 & & & & & & & ------ & 68.9 & 78.1 & 90.0 & 98.4 & 67.6 \\
\hline mGBP7 & & & & & & & & ------ & 60.9 & 68.8 & 68.6 & 52.3 \\
\hline mGBP8 & & & & & & & & & ------ & 90.2 & 91.3 & 65.8 \\
\hline mGBP9 & & & & & & & & & & ----- & 88.7 & 66.5 \\
\hline mGBPIO & & & & & & & & & & & ----- & 67.3 \\
\hline mGBPI I & & & & & & & & & & & & ------ \\
\hline
\end{tabular}


often expressed in a tissue-specific manner [30]. Further studies will be necessary to verify a potential tissue-specific expression/regulation of the different mRNA isoforms of mGBP3 and mGBP4.

\section{Evolution of the $m G B P s$}

Recent data indicate that the GBPs are host effector molecules involved in pathogen defense $[5,16,17]$. Defense against pathogens requires permanent adaptation to the changes in pathogen virulence strategies [31,32]. We suggest that evolutionary pressure led to gene duplication events which have resulted in the current mGBP clusters on chromosomes 3 and 5. It is most likely, that these gene duplications started with one primordial mGBP. We suppose that this ancestor mGBP is located on chromosome 3 because these mGBPs are more divergent among each other as compared to the mGBPs on chromosome 5. Interestingly, on chromosome 3 two homology clusters have evolved. One homology cluster with $m G b p 1, m G b p 2$, and $m G b p 5$ is characterized by a C-terminal CaaX motif for isoprenylation and a 'TLRD' G4 motif. In contrast, the second homology cluster with $m G b p 3$ and $m G b p 7$ lacks the CaaX motif and possess a 'TVRD' G4 motif. This finding leads to the hypothesis, that $m \mathrm{~Gb} p 3$ or $m G b p 7$ is the ancestor for all mGBPs on chromosome 5 which also lack a CaaX motif and have a "TVRD" G4 motif. This is further corroborated by the high cDNA sequence identities (around 70\%) of mGBP3 and mGBP7 with mGBP4, mGBP6, mGBP8, mGBP9, mGBP10, and mGBP11 on chromosome 5. On this chromosome also the most recent duplication event occurred, where $m G b p 6$ emanated from $m \mathrm{Gbp} 10$ or vice versa. This is supported by the high homology of $98.4 \%$ between these two GTPases. Moreover, we suggest that the mGBP cluster on chromosome 5 is a "genomic hot spot" permanently exposed to genetic recombination events. Consistent with this suggestion, we detected a transposon-like element ( $>1000 \mathrm{bp}$ ) which is integrated several times in this mGBP cluster (data not shown). Further studies have to clarify whether these genes evolved due to evolutionary pressure and whether these genes have redundant or non-redundant functions during pathogen defense.

We have shown that mGBPs are highly induced upon IFN $\gamma$ stimulation and infection with intracellular bacteria or protozoa indicating an important role as effector molecules in host defense. Now, we describe and characterize the genomic loci of the mGBPs on chromosomes 3 and 5 . These data will be very important for the analyses of evolutionary gene amplifications required in host defense as well as for functional studies by the generation of gene targeted mice which are under way.

\section{Methods \\ Determination of the genomic sequences and localization of the $m G B P s$}

To analyse the chromosomal localization of the mGBPs the mouse genome research page on the Ensembl website http://www.ensembl.org, version 38 was used. The genomic sequences of the appropriate chromosomal segments were determined using bacterial artificial chromosome (BAC) sequences. To display all available BACs on the contig view the corresponding field was activated using the 'decorations' function. For this study we have chosen the sequences of the BAC clones RP23-100J23 and RP24-314I8 for chromosome 3 and RP24-63G23, RP23329M7, RP23-152O10, and RP24-210D14 for chromosome 5 . We obtained the BACs' genomic sequences from the website of the National Center for Biotechnology Information (NCBI) http://www.ncbi.nlm.nih.gov. The sequences were downloaded [Accession numbers: AC102108 (RP23-100J23), AC115865 (RP24-314I8), AC113980 (RP24-63G23), AC123697 (RP23-329M7), AC144914 (RP24-210D14), and AC162798 (RP23152O10)] and imported into EditSeq (DNAStar, Madison, WI) for assembly into contigs using the SeqMan II program (DNAStar, Madison, WI). For further analyses expressed sequence tags (ESTs) of mGBPs were obtained with the Basic Local Alignment Search Tool (BLAST) 'blastn' [25] from the NCBI website and mapped onto the respective genomic area.

\section{Identification of the exon-intron structure}

The transcript sequences from mGBP4 (mpa-2) and mGBP6 (mpa-2l) were downloaded from the Ensembl website and imported into EditSeq. We then determined the exon-intron structure by using BLAST "align two sequences" using standard parameters on the NCBI website. The sequences of single exons from mGBP4 and mGBP6 were aligned with the corresponding BAC sequences. If the exons mapped to multiple regions on the BAC with high homology the regions were retained and imported into EditSeq. The equivalent sequences from the resulting BLAST hits on the BACs were subsequently aligned with MeqAlign (DNAStar, Madison, WI) using standard parameters. The 3 ' and 5' splice sites were identified manually by inspecting the alignment and confirmed by comparing the genomic sequences to the corresponding exon sequences. Once all exons were mapped on the genome the exons indicating a new gene locus were assembled and potential cDNAs were created. Finally, we determined the open reading frames by using the ORF search tool from EditSeq.

\section{Alignment and phylogenetic tree \\ The alignment of mGBP cDNAs was created with the soft- ware ClustalW [33] and the subsequent layout was done with JalView http://www.jalview.org. The phylogenetic}


analysis was accomplished using the maximum likelihood method and treepuzzle http://www.tree-puzzle.de for construction of the phylogenetic tree. The treepuzzle software was run with the option for exact parameter estimates using the neighbor joining method. Finally, we used the software drawtree from the phylip package http:/ /www.phylip.com to plot the tree data. All software was run on a Linux PC workstation.

\section{Cell culture and stimulation}

The macrophage cell line ANA-1 [34] was cultured in very low endotoxin RPMI (Biochrom, Berlin, Germany) supplemented with $10 \%$ heat inactivated, low endotoxin fetal calf serum (Cambrex, Veniers, Belgium) and $50 \mu \mathrm{M} 2-\beta$ Mercaptoethanol (Invitrogen, Karlsruhe, Germany).

For stimulation of ANA-1 cells we used $100 \mathrm{U} / \mathrm{ml}$ recombinant mouse IFN $\gamma$ (R\&D Systems, Mainz, Germany). After $16 \mathrm{~h}$ of IFN $\gamma$ stimulation the cells were harvested for RNA preparation.

\section{Infection with Listeria monocytogenes}

C57BL/6N mice were purchased from Charles River (Sulzfeld, Germany) and maintained in the animal facility of the Medical Faculty of the Heinrich-Heine-University under SPF conditions. All procedures performed on animals in this study have been approved by the Animal Care and Use Committee of the local government of Duesseldorf and have been in accordance with the German animal laws. $\mathrm{C} 57 \mathrm{BL} / 6 \mathrm{~N}$ mice were intraperitoneally infected with $0.1 \times \mathrm{LD}_{50}$ L. monocytogenes (American type culture collection strain 43251), and organs were removed $48 \mathrm{~h}$ after infection.

\section{Amplification and cloning of $m G B P s$}

Total RNA from cells and tissues was isolated using Trizol Reagent (Invitrogen) according to the manufacture's instructions. First-strand cDNA synthesis was performed using $1 \mu \mathrm{g}$ of total RNA with M-MLV reverse transcriptase and oligo dT primer (Invitrogen). The subsequent PCR reactions were accomplished using specific forward and reverse primers (additional file 3), and sequencing for both DNA strands was done by GATC Biotech AG (Konstanz, Germany).

\section{Accession numbers of EST and CDNA sequences}

NCBI accession numbers of EST and CDNA sequences for all mGBPs are listed. The cDNA sequence numbers are shown in bold. In the case of mGBP6 and mGBP10 some ESTs could not be assigned to one individual GBP due to sequence identities and are therefore grouped together.
mGBP-I

$\begin{array}{llll}\text { AW476703, } & \text { BB033108, } & \text { BI853322, } & \text { BQ126171, } \\ \text { BY575536, CA894196, } & \text { CJ141369, } & \text { EF494422 }\end{array}$ NM_010259

mGBP-2

AV337765, BB840634, BI249610, BI790719, BM933217, BM935622, BQ550593, BQ550594, CB574409, CF583081, CF583082, CJ055311, CJ056573, CJ057752, NM_010260

\section{$m G B P 3$}

AA162247, AA170007, AA276469, AA276918, AA289618, AA289780, AA839370, AW228655, BB862339, BE282356, BE847132, BG861498, BG861928, BG862944, BG864332, BG865051, BG914515, BG915574, BG973623, BI105618, BI653801, BI653963, BI654072, BI654363, BI656878, BI656930, BI567265, BI658478, BI661510, BM120304, BM206838, BM210659, BM221385, BM222892, BM240875, BM244805, BP760734, BQ552945, BQ552946, BX528767, BY215748, BY329622, BY483332, BY487480, BY562117, CA541279, CA544763, CA546244, CA573999, CA577912, CF910901, CK329532, CK331660， CK389037， CX205022， NM_018734, U44731, BC019195

mGBP4

BB859245, BI661547, BY225616, CJ141442, CJ141546, EF494424, NM_008620

mGBP4.I

BF138720, BI659458, BY749043, EF494423

$m G B P 4+m G B P 4 . I$

AA170248, AA866719, AW228052, BE227153, BE336008, BI659458, BG864747, BG865054, BM244843, BM244986, CF911085

$m G B P 5$

AI021670, AV329198, BB022173, BB617729, BB634786, BF163382, BG863163, BI558563, BI662495, BY212549, BY214522, BY220452, BY221363, BY224819, NM_153564

mGBP6

AK128993, BC057969, BC115768, BF015742, BF015763, BK005759

mGBPIO

BI853721, BG915468, BI853566, DQ985743

$m G B P 6+m G B P I 0$

AA140542, AA267762, BE687038, BG915105, BG916153, BG916251， BM239887, BM241485, 
$\begin{array}{llll}\text { BM242358, } & \text { BM243209, } & \text { BP764987, } & \text { BX525828, } \\ \text { BY765347, } & \text { CA572886, } & \text { CD351560, } & \text { CJ062488, }\end{array}$ CK331259, CN660654

$m G B P 7$

AA200741, AI226719, AW106727, BB554853, BB666410, BF452604, BG914537, BI657558, BK005760, BP766023, BX513589, BX522404, BY016011, BY178861, BY197520, BY215691, BY221698, BY221980, BY223566BY742706, BY 761161, BY761419, BY765425, BY765812, CA574325, CA576100, CA885708, CF899303, CJ137202, CJ140108, CK342680, CK342922, CK343278, CX207094, CX219108, DV057094

\section{$m G B P 8$}

AA155498, BE686748, BM245207, BM245316, BM246467, BQ552741, DQ295175, BY717902, BI657260, CJ141546, CJ141512, NM_029509

\section{$m G B P 9$}

AA122564, BE650518, BE692183, BE849117, BG915970, BY747136, CA535632, CJ165022, CJ183419, CJ164864， BY060909， BY064961， DQ985742, NM_172777

mGBPI I

AA087907, BC111039, BI145047, BI655333, BF470780, BI660636

\section{Availability and requirements}

To analyse the chromosomal localization of the mGBPs the mouse genome research page on the Ensembl website http://www.ensembl.org/Multi/newsview?rel=38 was used. We obtained the BACs' genomic sequences from the website of the National Center for Biotechnology Information (NCBI) http://www.ncbi.nlm.nih.gov. The alignment of mGBP cDNAs was created with the software ClustalW [33] and the subsequent layout was done with JalView http://www.jalview.org. The phylogenetic analysis was accomplished using the maximum likelihood method and treepuzzle http://www.tree-puzzle.de for construction of the phylogenetic tree. The treepuzzle software was run with the option for exact parameter estimates using the neighbor joining method. Finally, we used the software drawtree from the phylip package http:/ \www.phylip.com to plot the tree data. All software was run on a Linux PC workstation.

\section{Authors' contributions}

AK carried out the in silico analysis and drafted the manuscript. CK performed the PCRs and drafted the manuscript. JW participated in the sequence alignment and generated the phylogenetic tree. CBG carried out the infection experiments and stimulation of macrophages and prepared all cDNA. DD participated in the sequence align- ment and drafted the manuscript. SB participated in the design of the study and drafted the manuscript. KP conceived the study, and participated in its design and coordination. All authors read and approved the final manuscript.

\section{Additional material}

\section{Additional file 1}

ClustalW alignment of predicted protein sequences of the eleven $65 \mathrm{kDa}$ $m \mathrm{GBP}$ members. The alignment-layout was edited with the JalView software. Amino acids identical in all eleven $m G B P$ s are marked in dark blue, identical in $\geq 75 \%$ in medium blue and identical in $\geq 50 \%$ in light blue. Red boxes highlight the conserved GTP binding motifs: P-loop (G1),

Switch I (G2), Switch II (G3) and G4. The green box highlights the conserved CaaX motif.

Click here for file

[http://www.biomedcentral.com/content/supplementary/1471-

2164-9-158-S1.eps]

\section{Additional file 2}

ClustalW alignment of ORF sequences of the eleven $65 \mathrm{kDa} \mathrm{mGBP}$ members. The alignment-layout was edited with the JalView software. Basepairs identical in all eleven $m$ GBPs are marked in dark blue, identical in $\geq 75 \%$ in medium blue and identical in $\geq 50 \%$ in light blue. Red boxes highlight the conserved GTP binding motifs: P-loop (G1), Switch I (G2), Switch II (G3) and G4.

Click here for file

[http://www.biomedcentral.com/content/supplementary/1471-

2164-9-158-S2.tiff]

\section{Additional file 3}

Primer pairs used for amplification of $m G B P 6, m G B P 7, m G B P 8$, $m G B P 9, m G B P 10$, and $m G B P 11$. Due to the high homology an identical forward primer for $m G B P 8, m G B P 9$ and $m G B P 11$ combined with specific reverse primers were used. For $m G B P 6$ and $m G B P 10$ an identical reverse primer and specific forward primers which bind in the 5' UTR were used. GAPDH served as an internal control.

Click here for file

[http://www.biomedcentral.com/content/supplementary/1471-

2164-9-158-S3.doc]

\section{Acknowledgements}

We thank Gerrit Praefcke for helpful discussions. This work was supported by grants PF259/3-3, FOR729 and Leibniz (to K.P.) and the GRK 1045/I (to S.B. and K.P.) of the Deutsche Forschungsgemeinschaft (DFG) and MUGEN (to K.P.). The authors declare that they have no financial conflict of interests that might be construed to influence the results or interpretation of the manuscript.

\section{References}

I. Gupta SL, Rubin BY, Holmes SL: Interferon action: induction of specific proteins in mouse and human cells by homologous interferons. Proc Natl Acad Sci U S A 1979, 76:4817-482I.

2. Cheng YS, Colonno RJ, Yin FH: Interferon induction of fibroblast proteins with guanylate binding activity. J Biol Chem 1983, 258:7746-7750.

3. Decker T, Lew DJ, Cheng YS, Levy DE, Darnell JE Jr.: Interactions of alpha- and gamma-interferon in the transcriptional regu- 
lation of the gene encoding a guanylate-binding protein. $E M B O$ J 1989, 8:2009-2014

4. Decker T, Lew DJ, Darnell JE Jr.: Two distinct alpha-interferondependent signal transduction pathways may contribute to activation of transcription of the guanylate-binding protein gene. Mol Cell Biol I99I, I I:5 I47-5I53.

5. Degrandi D, Konermann C, Beuter-Gunia C, Kresse A, Wurthner Kurig S, Beer S, Pfeffer K: Extensive Characterization of IFNInduced GTPases mGBPI to mGBPIO Involved in Host Defense. J Immunol 2007, I 79:7729-7740.

6. Lew DJ, Decker T, Strehlow I, Darnell JE: Overlapping elements in the guanylate-binding protein gene promoter mediate transcriptional induction by alpha and gamma interferons. Mol Cell Biol 1991, I I:182-191.

7. Guenzi E, Topolt K, Cornali E, Lubeseder-Martellato C, Jorg A Matzen K, Zietz C, Kremmer E, Nappi F, Schwemmle M, Hohenadl C Barillari G, Tschachler E, Monini P, Ensoli B, Sturzl M: The helical domain of GBP-I mediates the inhibition of endothelial cell proliferation by inflammatory cytokines. EMBO J 200I, 20:5568-5577.

8. Lubeseder-Martellato C, Guenzi E, Jorg A, Topolt K, Naschberger E, Kremmer E, Zietz C, Tschachler E, Hutzler P, Schwemmle M, Matzen K, Grimm T, Ensoli B, Sturzl M: Guanylate-binding proteinexpression is selectively induced by inflammatory cytokines and is an activation marker of endothelial cells during inflammatory diseases. Am J Pathol 2002, I6I:1749-1759.

9. Schwemmle M, Staeheli P: The interferon-induced 67-kDa guanylate-binding protein (hGBPI) is a GTPase that converts GTP to GMP. J Biol Chem 1994, 269: | | 299-I | 305

10. Praefcke GJ, Geyer M, Schwemmle M, Robert KH, Herrmann C: Nucleotide-binding characteristics of human guanylate-binding protein I (hGBPI) and identification of the third GTP binding motif. J Mol Biol I 999, 292:32 I-332.

II. Prakash B, Praefcke G], Renault L, Wittinghofer A, Herrmann C: Structure of human guanylate-binding protein I representing a unique class of GTP-binding proteins. Nature 2000, 403:567-57l.

12. Praefcke GJ, McMahon HT: The dynamin superfamily: universal membrane tubulation and fission molecules? Nat Rev Mol Cell Biol 2004, 5: 133-147.

13. Gorbacheva VY, Lindner D, Sen GC, Vestal DJ: The interferon (IFN)-induced GTPase, mGBP-2. Role in IFN-gammainduced murine fibroblast proliferation. J Biol Chem 2002, 277:6080-6087.

14. Guenzi E, Topolt K, Lubeseder-Martellato C, Jorg A, Naschberger E, Benelli R, Albini A, Sturzl M: The guanylate binding protein-I GTPase controls the invasive and angiogenic capability of endothelial cells through inhibition of MMP-I expression. EMBO J 2003, 22:3772-3782.

15. Duan Z, Foster R, Brakora KA, Yusuf RZ, Seiden MV: GBPI overexpression is associated with a paclitaxel resistance phenotype. Cancer Chemother Pharmacol 2006, 57:25-33.

16. Anderson SL, Carton JM, Lou J, Xing L, Rubin BY: Interferoninduced guanylate binding protein-I (GBP-I) mediates an antiviral effect against vesicular stomatitis virus and encephalomyocarditis virus. Virology 1999, 256:8-14.

17. Carter CC, Gorbacheva VY, Vestal DJ: Inhibition of VSV and EMCV replication by the interferon-induced GTPase, mGBP-2: differential requirement for wild-type GTP binding domain. Arch Virol 2005, 150:1213-1220.

18. Asundi VK, Stahl RC, Showalter L, Conner KJ, Carey DJ: Molecular cloning and characterization of an isoprenylated $67 \mathrm{kDa}$ protein. Biochim Biophys Acta 1994, I 2 1 7:257-265.

19. Schwemmle M, Kaspers B, Irion A, Staeheli P, Schultz U: Chicken guanylate-binding protein. Conservation of GTPase activity and induction by cytokines. J Biol Chem 1996, 27 I: 10304-10308.

20. Robertsen B, Zou J, Secombes C, Leong JA: Molecular and expression analysis of an interferon-gamma-inducible guanylatebinding protein from rainbow trout (Oncorhynchus mykiss). Dev Comp Immunol 2006, 30:1023-1033.

21. Olszewski MA, Gray J, Vestal DJ: In silico genomic analysis of the human and murine guanylate-binding protein (GBP) gene clusters. J Interferon Cytokine Res 2006, 26:328-352.

22. Tripal P, Bauer M, Naschberger E, Mortinger T, Hohenadl C, Cornali $E$, Thurau M, Sturzl M: Unique features of different members of the human guanylate-binding protein family. I Interferon Cytokine Res 2007, 27:44-52.

23. Boehm U, Guethlein L, Klamp T, Ozbek K, Schaub A, Futterer A, Pfeffer K, Howard JC: Two families of GTPases dominate the complex cellular response to IFN-gamma. J Immunol 1998, 161:67|5-6723.

24. Nguyen TT, Hu Y, Widney DP, Mar RA, Smith JB: Murine GBP-5, a new member of the murine guanylate-binding protein family, is coordinately regulated with other GBPs in vivo and in vitro. J Interferon Cytokine Res 2002, 22:899-909.

25. Altschul SF, Gish W, Miller W, Myers EW, Lipman DJ: Basic local alignment search tool. I Mol Biol 1990, 21 5:403-4IO.

26. Konermann C, Kresse A, Beuter-Gunia C, Wurthner J, Degrandi D, Pfeffer K, Beer S: In Silico and In Vitro Characterization of mGBP4 Splice Variants. DNA Cell Biol 2007.

27. Vestal DJ, Gorbacheva VY, Sen GC: Different subcellular localizations for the related interferon-induced GTPases, MuGBP-I and MuGBP-2: implications for different functions? J Interferon Cytokine Res 2000, 20:99I-1000.

28. Shenoy AR, Kim BH, Choi HP, Matsuzawa T, Tiwari S, MacMicking JD: Emerging themes in IFN-gamma-induced macrophage immunity by the p47 and p65 GTPase families. Immunobiology 2007, 2 I 2:77|-784

29. Hughes TA: Regulation of gene expression by alternative untranslated regions. Trends Genet 2006, 22: I 19-122.

30. Zhang $T$, Haws $P, W u$ Q: Multiple variable first exons: a mechanism for cell- and tissue-specific gene regulation. Genome Res 2004, I 4:79-89.

31. Hedrick SM: The acquired immune system: a vantage from beneath. Immunity 2004, 21:607-615.

32. Trowsdale J, Parham P: Mini-review: defense strategies and immunity-related genes. Eur J Immunol 2004, 34:7-I7.

33. Thompson JD, Higgins DG, Gibson TJ: CLUSTAL W: improving the sensitivity of progressive multiple sequence alignment through sequence weighting, position-specific gap penalties and weight matrix choice. Nucleic Acids Res 1994, 22:4673-4680.

34. Cox GW, Mathieson BJ, Gandino L, Blasi E, Radzioch D, Varesio L: Heterogeneity of hematopoietic cells immortalized by $v$ myc/v-raf recombinant retrovirus infection of bone marrow or fetal liver. J Natl Cancer Inst 1989, 8 I: I 492-I 496.

Publish with Bio Med Central and every scientist can read your work free of charge

"BioMed Central will be the most significant development for disseminating the results of biomedical research in our lifetime. "

Sir Paul Nurse, Cancer Research UK

Your research papers will be:

- available free of charge to the entire biomedical community

- peer reviewed and published immediately upon acceptance

- cited in PubMed and archived on PubMed Central

- yours - you keep the copyright 